\title{
Ranibizumab in the treatment of choroidal neovascularization on the border of an inferior staphyloma associated with tilted disc syndrome
}

This article was published in the following Dove Press journal:

Clinical Ophthalmology

29 March 2010

Number of times this article has been viewed

\author{
Luis Arias ${ }^{1,2}$ \\ Jordi Monés ${ }^{2}$ \\ 'Hospital Universitari de Bellvitge, \\ L'Hospitalet de Llobregat, Barcelona; \\ ${ }^{2}$ Institut de la Màcula i de la Retina, \\ Centro Médico Teknon, Barcelona
}

Correspondence: Luis Arias Department of Ophthalmology, Hospital Universitari de Bellvitge, C/Feixa Llarga, sn, 08907 - L'Hospitalet de Llobregat,

Barcelona, Spain

Tel +34 620898469

Fax +34 93260798 I

Email luisarias@telefonica.net
Purpose: To describe a case of choroidal neovascularization $(\mathrm{CNV})$ on the border of an inferior staphyloma associated with tilted disc syndrome treated with intravitreal ranibizumab.

Patients: Observational case report.

Methods: A patient with CNV on the border of an inferior staphyloma associated with tilted disc syndrome was imaged using fluorescein angiography, autofluorescence and spectral domain optical coherence tomography, and treated with intravitreal injections of ranibizumab.

Results: The patient received 3 ranibizumab injections during the 9-month follow-up. The visual acuity improved from 20/40 to 20/32 and the foveal thickness reduced from 470 microns to 248 microns. The angiograms showed resolution of leakage associated with CNV. There were no adverse events.

Conclusion: Intravitreal ranibizumab is an efficacious and safe treatment in the management of choroidal neovascularization on the border of an inferior staphyloma associated with tilted disc syndrome.

Keywords: intravitreal ranibizumab, choroidal neovascularization, tilted disc syndrome, inferior staphyloma

\section{Introduction}

The main features that characterize eyes with tilted disc syndrome are oval optic disc with oblique axis, inferonasal crescent, situs inversus of the retinal vessels, myopic astigmatism and visual field defects..$^{1-3}$ In addition, an inferior staphyloma is often associated with this syndrome and severe complications such as choroidal neovascularization (CNV) can occur when the border of the staphyloma lies across the macula. ${ }^{4-7}$ This CNV usually shows a classic pattern on fluorescein angiography (FA) resulting in significant visual loss.

Ranibizumab (Lucentis ${ }^{\circledR}$; Genentech Inc, South San Francisco, California, USA) is a humanized antibody fragment designed to bind and inhibit all vascular endothelial growth factor-A (VEGF-A) isoforms. It was approved by the Food and Drug Administration (FDA) for the treatment of wet age-related macular degeneration in June 2006. It has shown a great efficacy in the management of all angiographic subtypes of lesions in different clinical trials. ${ }^{8,9}$

We present a case of CNV on the border of an inferior staphyloma associated with tilted disc syndrome successfully treated with intravitreal ranibizumab.

\section{Case report}

A 77-year-old woman complained about visual acuity (VA) loss in her left eye in the previous 3 weeks. She had an uncomplicated cataract surgery in both eyes 4 years 
before. The patient did not have high myopia. The refractive error prior to cataract surgery was unknown since the patient was operated on in another hospital. However, the patient told us that she began to wear glasses when she was 45 years old.
At the moment of the examination her refractive error was $90^{\circ}-1.00+0.25$ in her right eye and $90^{\circ}-0.75+0.50$ in her left eye. The axial length was $22.9 \mathrm{~mm}$ in the right eye and $23.2 \mathrm{~mm}$ in the left eye. Her VA measured with ETDRS a)

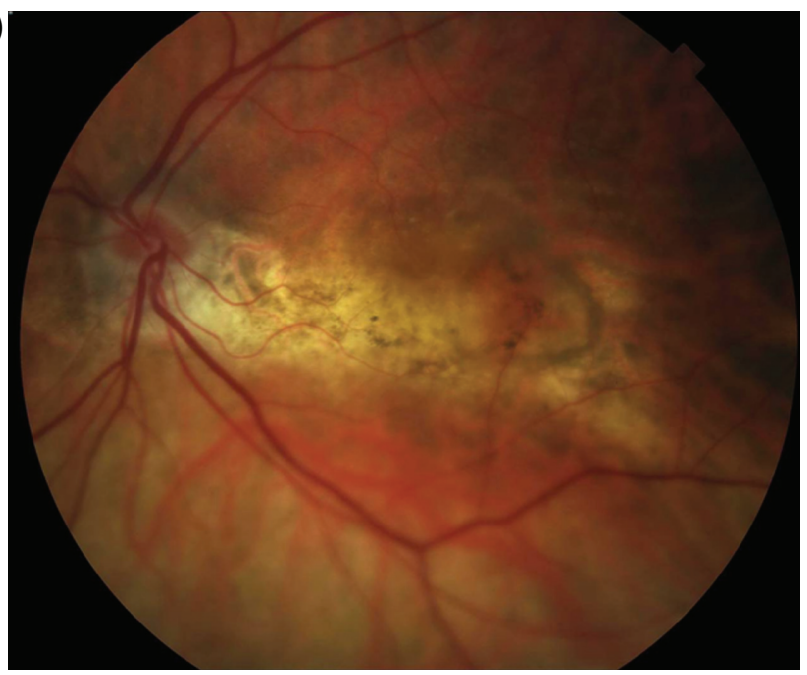

c)

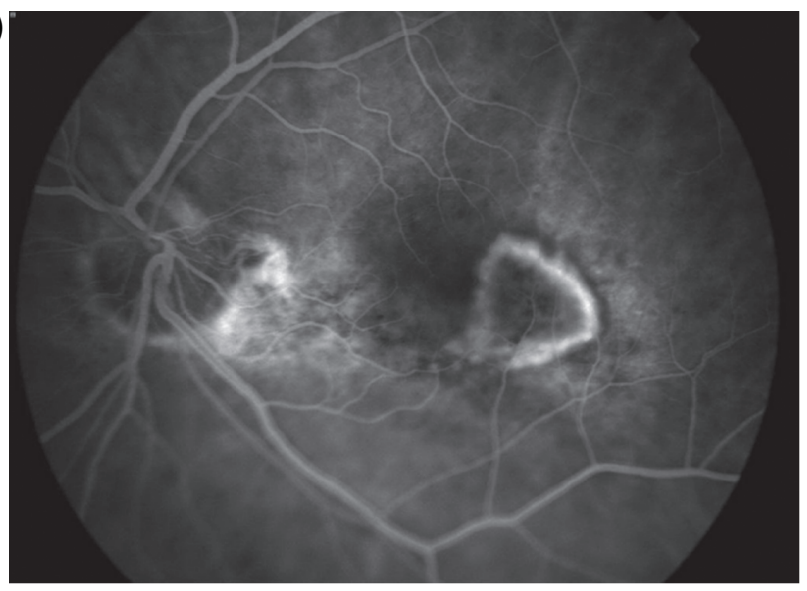

e)

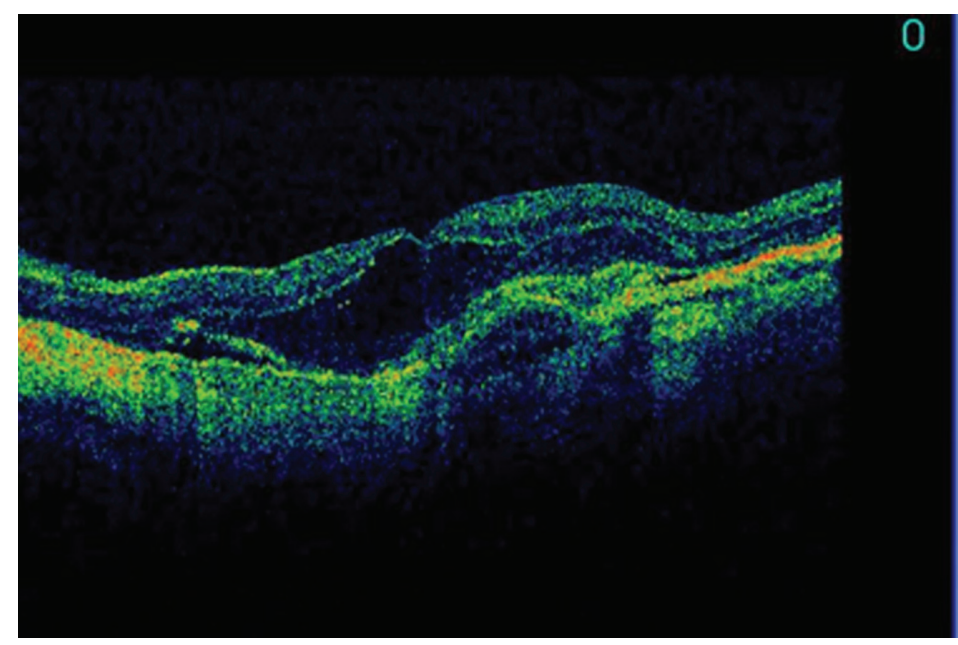

b)

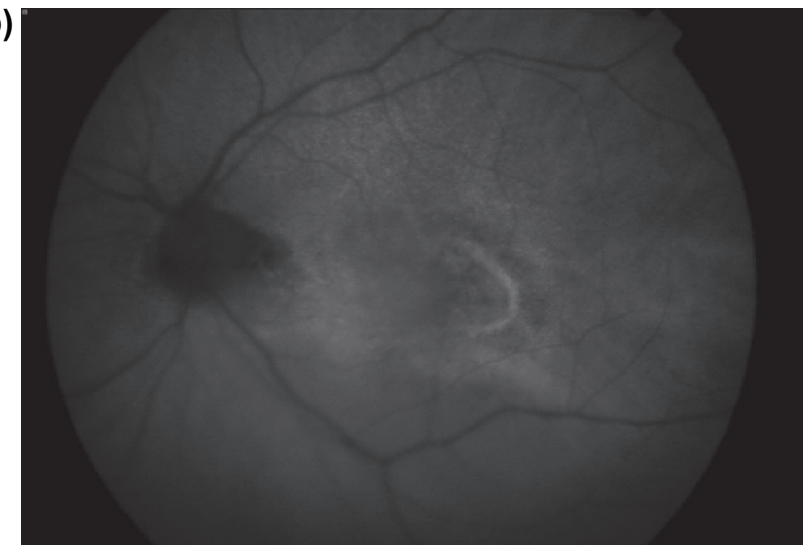

d)

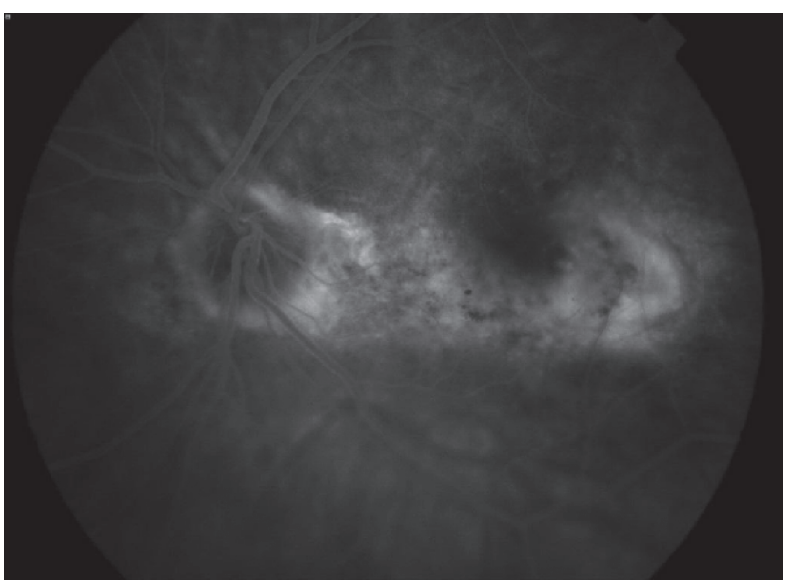

0

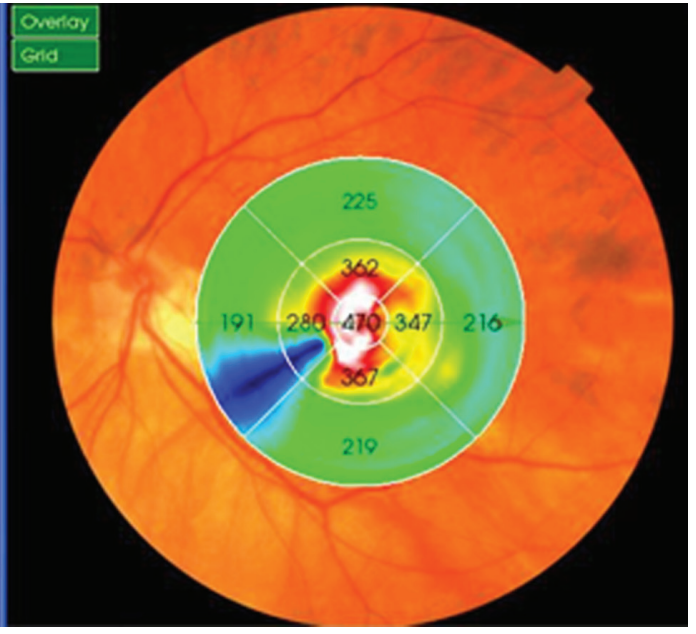

Figure I At baseline, the fundus photograph of the left eye revealed a tilted disc and the border of an inferior staphyloma lying across the macula and a subretinal lesion (a). Autofluorescence image showed mild hyperautofluorescence at the temporal border of the macula (b). Fluorescein angiography showed a well-defined predominantly classic choroidal neovascularization (CNV) in an early phase with leakage in a late phase (c, d). Spectral domain optical coherence tomography scanning showed an irregular and elevated retinal pigment epithelium band with overlying CNV associated with intraretinal and subretinal fluid (e). 
("Early Treatment of Diabetic Retinopathy Study") charts was 20/20 in the right eye and 20/40 in the left eye. The anterior segment examination showed transparent corneas and clear intraocular lenses located in the posterior chamber. The intraocular pressure was $16 \mathrm{mmHg}$ in both eyes. The fundus examination revealed tilted discs and the border of an inferior staphyloma lying across the maculas in both eyes. Imaging with FA (Imagenet ${ }^{\circledR}$; Topcon Corporation, Tokyo, Japan) showed an active predominantly classic CNV and a window defect on the border of the staphyloma. OCT scanning (OCT 3-D, Topcon Corporation, Tokyo, Japan) showed an irregular and elevated retinal pigment epithelium (RPE) band with a)

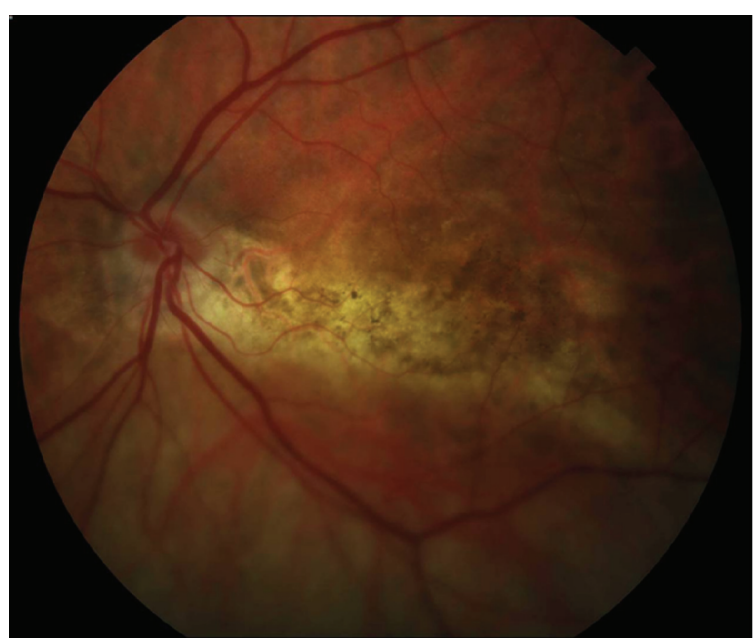

c)

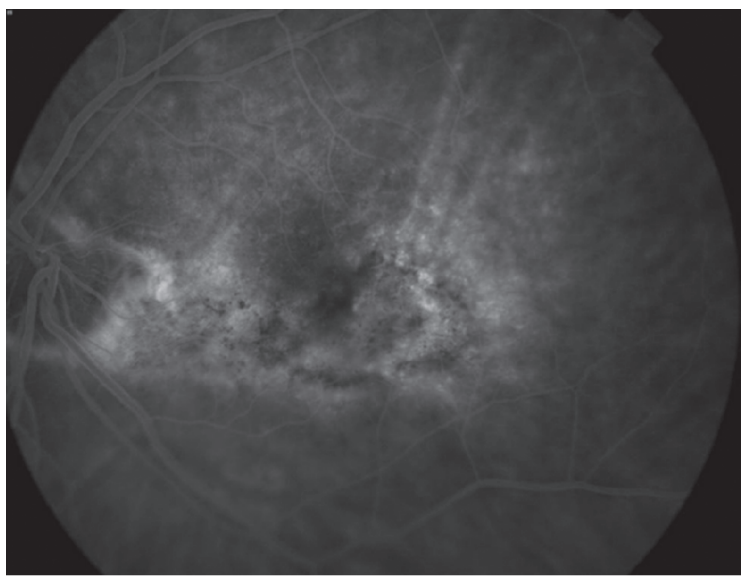

b)

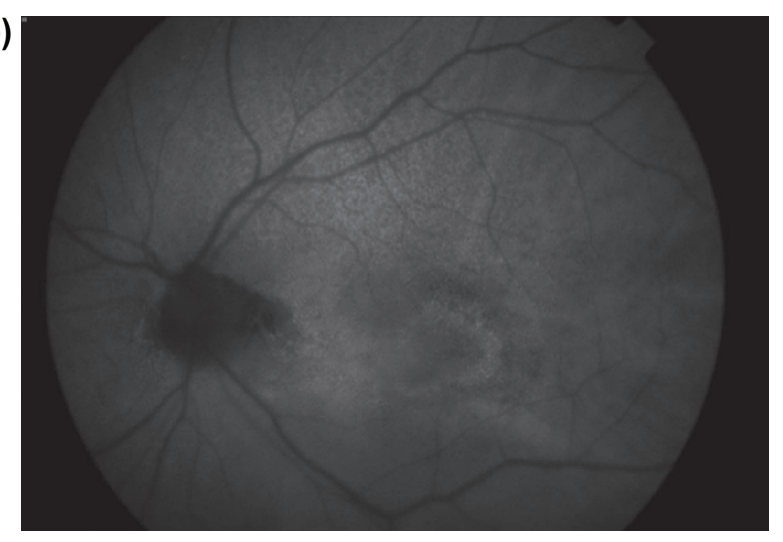

d)

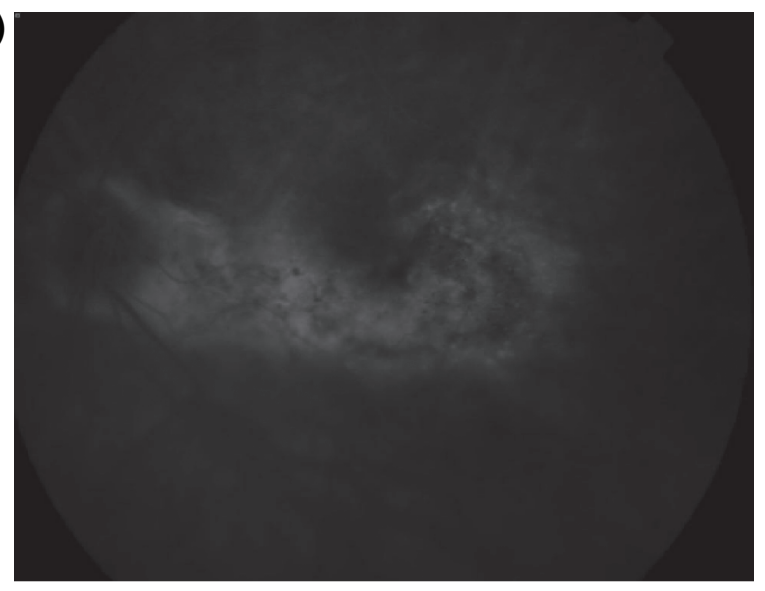

e)
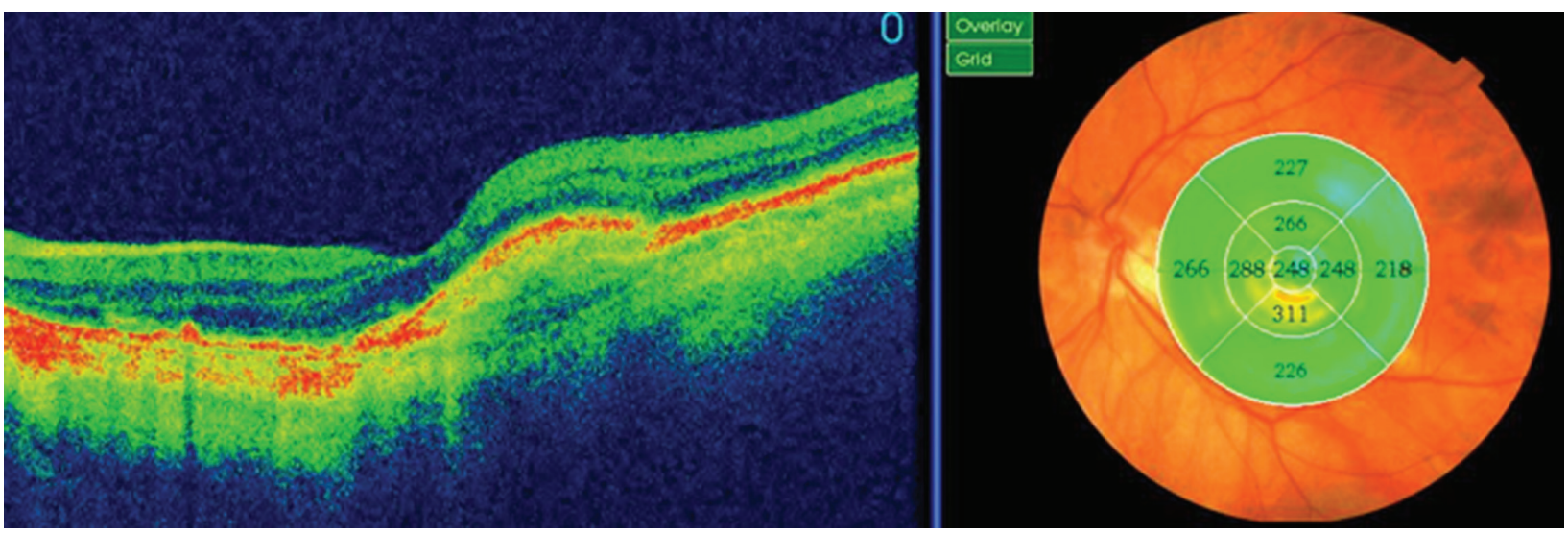

Figure 2 After three intravitreal injections of ranibizumab, the fundus photograph of the left eye revealed a flat macula with pigmentary changes (a). Autofluorescence image showed mild hyperautofluorescence at the temporal border of the macula (b). Fluorescein angiography showed staining but no leakage from the choroidal neovascular membrane at the early phase and the late phase as well $(\mathbf{c}, \mathbf{d})$. Spectral domain optical coherence tomography scanning showed no intraretinal or subretinal fluid with restoration of the foveal contour (e). 
overlying CNV associated with intraretinal and subretinal fluid (Figure 1). The patient was informed about her disease and the risks and benefits of the treatment. An informed consent was obtained. We administered three monthly intravitreal injections of ranibizumab $(0.5 \mathrm{mg} / 0.05 \mathrm{~mL})$ in the office under sterile conditions. The VA before CNV development was not available. After the first injection, the VA improved from 20/40 to 20/32. No further improvement was observed after the second and the third injection. The angiograms showed resolution of leakage associated with CNV. OCT scanning showed no intraretinal or subretinal fluid with restoration of the foveal contour (Figure 2). The foveal thickness reduced from 470 microns to 248 microns. There were no adverse events. The VA and the angiograms and OCT findings remained unchanged during the 9-month follow-up with no further treatment.

\section{Discussion}

Severe visual loss has been reported on eyes with tilted disc syndrome when the upper-temporal border of an inferior staphyloma lies across the macula. It has been hypothesized that the sharp curve on the border of the staphyloma causes mechanical or hemodynamic changes and promotes the development of macular complications. ${ }^{2}$ Several published papers have described CNV in these cases. ${ }^{5-7}$ Recently, Nakanishi et $\mathrm{al}^{4}$ reviewed 32 eyes of 20 patients with tilted disc syndrome and inferior staphyloma lying across the macula. They found macular complications in $78 \%$ of the patients consisting of polypoidal choroidal vasculopathy (PCV) (22\%), classic CNV (3\%), focal serous retinal detachment without PCV or CNV (41\%) and atrophy of the RPE alone (13\%). The VA of eyes with PCV or CNV was significantly worse than in the other cases. However, they did not report the long-term evolution and visual outcome and the proposed treatment.

We decided to treat our patient with intravitreal ranibizumab with the recommended standard protocol of treatment. ${ }^{10}$ Ranibizumab is indicated for choroidal neovascular lesions with active disease with three initial consecutive monthly injections (loading dose) and thereafter monthly monitoring based on VA, fundus examination and OCT findings. This flexible strategy of treatment is effective and more feasible than continued monthly injections used in pivotal clinical trials. ${ }^{8,9}$ In our case, VA improved with resolution of angiographic leakage and OCT scanning edema. After the loading dose, our patient did not require any additional injection during the 9-month follow-up period. Nevertheless, it is likely that further treatment will be required with longer follow-up. We did not have any ocular or systemic side effect related to the drug or the route of administration.

The VA improved by one line, from $20 / 40$ to $20 / 32$. In comparison, a much more substantial anatomical benefit was observed, with resolution of the angiographic leakage and the intraretinal and subretinal fluid detected on OCT scans. However, it is known that visual gaining is often limited in predominantly classic lesions growing between the photoreceptors and the RPE.

Photodynamic therapy with verteporfin could have been used as an alternative treatment in this patient. However, potential RPE damage could have limited visual recovery. ${ }^{11}$ Bevacizumab could have been used as an alternative treatment. However, it is not an approved drug for intraocular use in Spain. RPE rips have been reported with ranibizumab and bevacizumab as well. To our knowledge, no data support reduced risk of RPE rips with either ranibizumab or bevacizumab.

In summary, ranibizumab therapy can be used as an efficacious and safe treatment in the management of $\mathrm{CNV}$ associated with tilted disc syndrome. Nevertheless, this is only supported by a single case report. It would be desirable to accumulate more data to demonstrate potential safety and efficacy of ranibizumab in this condition.

\section{Disclosures}

The authors have no financial or proprietary interest in any product mentioned in this article, and received no financial support.

Dr Arias had full access to all the data in the study and takes responsibility for the integrity of the data and the accuracy of the data analysis as well as the decision to submit for publication.

\section{References}

1. Apple DJ, Rabb MF, Walsh PM. Congenital anomalies of the optic disc. Surv Ophthalmol. 1982;27:3-41.

2. Curtin BJ. The posterior staphyloma of pathologic myopia. Trans Am Ophthalmol Soc. 1977;75:67-86.

3. Young SE, Walsh FB, Knox DL. The tilted disk syndrome. Am JOphthalmol. 1976;82:16-23.

4. Nakanishi H, Tsujikawa A, Gotoh N, et al. Macular complications on the border of an inferior staphyloma associated with tilted disc syndrome. Retina. 2008;28:1493-1501.

5. Prost M, De Laey JJ. Choroidal neovascularization in tilted disc syndrome. Int Ophthalmol. 1988;12:131-135.

6. Stur M. Congenital tilted disk syndrome associated with parafoveal subretinal neovascularization. Am J Ophthalmol. 1988; 105:98-99. 
7. Tsuboi S, Uchihori Y, Manabe R. Subretinal neovascularisation in eyes with localised inferior posterior staphylomas. Br J Ophthalmol. 1984;68:869-872.

8. Rosenfeld PJ, Brown DM, Heier JS, et al. Ranibizumab for neovascular age-related macular degeneration. N Engl J Med. 2006;355:1419-1431.

9. Brown DM, Kaiser PK, Michels M, et al. Ranibizumab versus verteporfin for neovascular age-related macular degeneration. $N$ Engl J Med. 2006;355:1432-1444.
10. Mitchell P, Korobelnik JF, Lanzetta P, et al. Ranibizumab (Lucentis) in neovascular age-related macular degeneration: evidence from clinical trials. Br J Ophthalmol. 2009; [Epub ahead of print]. DOI:10.1136/ bjo.2009.159160.

11. Odergren A, Ming Y, Kvanta A. Photodynamic therapy of experimental choroidal neovascularisation in the mouse. Curr Eye Res. 2006;31: $765-774$.

\section{Publish your work in this journal}

Clinical Ophthalmology is an international, peer-reviewed journal covering all subspecialties within ophthalmology. Key topics include: Optometry; Visual science; Pharmacology and drug therapy in eye diseases; Basic Sciences; Primary and Secondary eye care; Patien Safety and Quality of Care Improvements. This journal is indexed on

Submit your manuscript here: http://www.dovepress.com/clinical-ophthalmology-journal

\section{Dovepress}

PubMed Central and CAS, and is the official journal of The Society of Clinical Ophthalmology (SCO). The manuscript management system is completely online and includes a very quick and fair peer-review system, which is all easy to use. Visit http://www.dovepress.com/ testimonials.php to read real quotes from published authors. 